\title{
Sustainable Transparent Conducting Oxide Nanomaterials; Aluminium- and Gallium-Co-Doped Zinc Oxide (AGZO)
}

\author{
Dougal P. Howard, Peter Marchand, Tharan Gordon, and Jawwad A. Darr* \\ Clean Materials Technology Group, Department of Chemistry, University College London, London WC1H OAJ, United Kingdom
}

\begin{abstract}
Transparent conducting oxide (TCO) nanoparticles based on zinc oxide doped with aluminium and gallium (Al- and Ga-co-doped ZnO, AGZO) were synthesized using a continuous hydrothermal flow synthesis (CHFS) reactor by rapid hydrolysis and dehydration of aqueous metal salt precursors, in the presence of formic acid as an in-process reducing agent. The as-synthesised powders were characterized by powder X-ray diffraction ( $p X R D$ ), transmission electron microscopy (TEM), scanning electron microscopy (SEM), energy dispersive X-ray spectroscopy (EDS) and X-ray photoelectron spectroscopy (XPS). The as-synthesised powders were pressed into ceramic compacts and heat treated to ensure phase-pure wurtzite structure. The electrical properties of the materials were evaluated by Hall Probe measurements on the compacts, showing resistivities as low as $4.55 \times 10^{-2} \Omega \mathrm{cm}$, which represents a very high conductivity for such a pressed disc based on $\mathrm{ZnO}$.
\end{abstract}

\section{Keywords:}

Transparent conducting oxide (TCO) thin films have found many uses in modern electronic devices, particularly as a key component in touch screens, flat panel displays, solar cells, smart windows, and organic light emitting diodes (OLEDs). ${ }^{1}$ Indium tin oxide (ITO) is the current industrystandard TCO material used in such devices due to its high optical transparency $(>80 \%)$ and low resistivities (order of $10^{-4} \Omega \mathrm{cm}$ ) when deposited as thin films by methods such as magnetron sputtering, ${ }^{2}$ pulsed laser deposition, ${ }^{3}$ spincoating, ${ }^{4}$ spray pyrolysis, ${ }^{5}$ and chemical vapor deposition. ${ }^{6}$

Due to the high cost and increasing scarcity of indium other more sustainable, non-toxic, and inexpensive TCO materials are being explored as potential ITO replacements. Such target TCO materials are being sought that possess a direct band gap $>3 \mathrm{eV}$, and transparency and resistivity figures which are comparable to those of ITO. ${ }^{7}$ Thin films of zinc oxide show high transparencies, but require metal-ion doping to approach the conductivity properties of ITO. $^{8}$ The two most widely studied dopants for the $\mathrm{ZnO}$ system are aluminium $\left(\mathrm{Al}^{3+}\right)$ and gallium $\left(\mathrm{Ga}^{3+}\right)$, which have achieved results rivalling ITO's optoelectronic properties. ${ }^{9,}{ }^{10}$ In comparison to ITO,

*Author to whom correspondence should be addressed. aluminium-doped $\mathrm{ZnO}(\mathrm{AZO})$ is cheaper by virtue of $\mathrm{Al}$ being used, however it suffers in terms of chemical stability, particularly with regards to water stability. Galliumdoped $\mathrm{ZnO}$ (GZO) is more costly due to the presence of $\mathrm{Ga}$, but is more stable to hydrolysis than AZO. ${ }^{11}$ Therefore, it has been suggested that $\mathrm{Al}$ and $\mathrm{Ga}$ co-doped into $\mathrm{ZnO}$ (AGZO) could offer a reasonable balance of stability, performance and cost for a sustainable $\mathrm{ZnO}$-based TCO. ${ }^{12}$ For highly transparent thin films deposited with various sputtering techniques, resistivities as low as 6 to $8 \times 10^{-4} \Omega \mathrm{cm}$ have been reported for AGZO. ${ }^{13,14} \mathrm{In}$ comparison to the number of reports in the TCO literature on AZO or GZO, the AGZO system is relatively under-explored.

TCO powders can be processed into thick or thin films via spin coating and inkjet methods, however subsequent annealing steps on the deposited films often require temperatures in excess of $700{ }^{\circ} \mathrm{C}$ to have adequate adhesion. ${ }^{15,16}$ This limits the appropriate choice of substrates to those that can withstand such temperatures. A wider field of substrates (e.g., flexible plastics ${ }^{17}$ ) can be considered when TCOs are deposited from suitable ceramic inks using ink-jet printing followed by rapid microwave annealing, for example. Therefore, there is 
interest to develop reproducible and sustainable manufacturing routes to stable and high loading TCO inks, which could be suitable for use in inkjet, screen printing or related printing methods.

Continuous hydrothermal flow synthesis (CHFS) reactors offer a scalable and sustainable process technology for the fabrication of nanoparticles as ceramic dispersions in water. The CHFS process uses superheated water and inexpensive metal salts, with $\mathrm{pH}$ modifiers, as reagents and can allow nanoparticles to be functionalized in-process, potentially aiding dispersion into a number of different solvent systems. ${ }^{18}$ In a CHFS reactor, nanoparticles rapidly form when a flow of supercritical (or superheated) water is mixed with an ambient temperature aqueous flow of metal salts (and other reagents, such as base, if needed) in an engineered mixer such as a confined jet mixer. By virtue of being a continuous process, CHFS lends itself to process intensification, which opens up the possibility of it being used for direct, scalable and continuous TCO-ceramic ink production. ${ }^{19-21}$ In CHFS, the mechanism of precursor conversion to nanoparticle metal oxide in the supercriti$\mathrm{cal} / \mathrm{superheated} \mathrm{water} \mathrm{environment} \mathrm{proceeds} \mathrm{via} \mathrm{simultane-}$ ous and rapid hydrolysis and dehydration. A schematic for the CHFS process is shown in Figure 1. After the mixer, the newly formed aqueous nanoparticle slurry is rapidly cooled in flow before passing through a back-pressure regulator valve, where it is collected for removal of the water and residual ions. ${ }^{19-22}$

To the knowledge of the authors, doped zinc oxide synthesis via CHFS for TCO applications has not previously been investigated, however, zinc oxides made via CHFS have been of interest for other applications on the laband pilot-scale ${ }^{20-22}$ this includes a combinatorial approach (lab-scale) to d- and f-block doped zinc oxides (0.5-1.5\%)

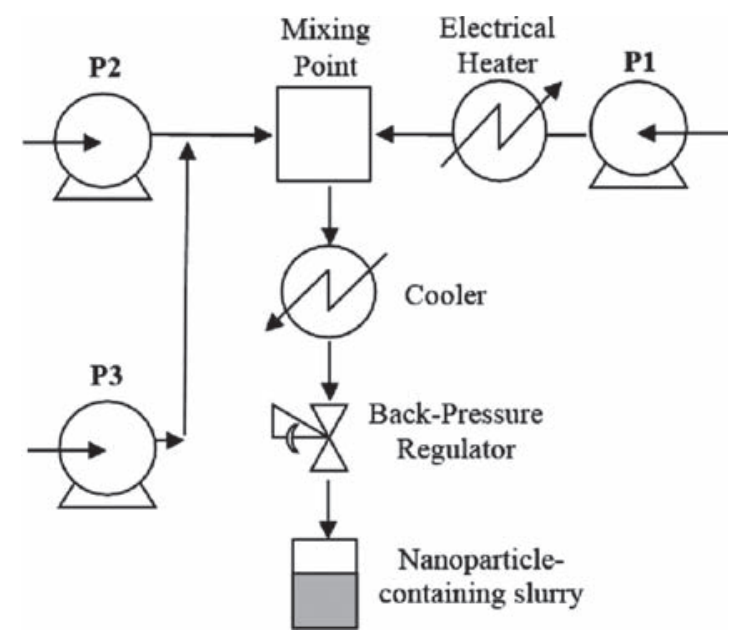

Figure 1. General schematic for the CHFS process; ambient precursor metal solution and base solution meet a stream of supercritical water, forming metal oxide nanoparticles which are collected as a slurry after cooling. for UV attenuation/photocatalysis and also $\mathrm{ZnO}$ made via a scaled-up pilot plant CHFS. ${ }^{20,21,23}$

Given the conduction mechanism for doped zinc oxides, which is based on intrinsic $\mathrm{H}$-impurities of the materials, ${ }^{24}$ reducing conditions in-process is assumed to be useful in the manufacture of $\mathrm{ZnO}$ for TCOs. In CHFS, formic acid has been used previously in-process to encourage the formation of oxygen vacancies in ITO nanoparticles for TCOs, ${ }^{18}$ as under such temperatures and pressures it breaks down to a mixture of reducing hydrogen and carbon dioxide according to Eq. (1). ${ }^{25}$ The vast excess of $\mathrm{H}_{2} \mathrm{O}$ in the reactor during the process firmly shifts the equilibrium to the right, ensuring the presence of $\mathrm{H}_{2}$ in the process. ${ }^{26,27}$

$$
\mathrm{H}_{2} \mathrm{O}+\mathrm{CO} \leftrightharpoons \mathrm{HCOOH} \leftrightharpoons \mathrm{CO}_{2}+\mathrm{H}_{2}
$$

Herein, we report the synthesis, characterisation, and electrical properties of aluminium and gallium co-doped zinc oxide by CHFS. After initial optimisation, the atomic ratio of $\mathrm{Zn}: \mathrm{Al}: \mathrm{Ga}$ used in the precursor solution was selected as 95:3:2.

Powder X-ray diffraction data of the as-prepared products, made with concentrations of formic acid from 0 to $0.75 \mathrm{M}$, (see Figs. 2(a-d)) confirmed the formation of wurtzite $\mathrm{ZnO}$, with peaks consistent with the included $\mathrm{ZnO}$ standard reference pattern (PDF No. 01-076-0704,

(a) $A G Z O(0)$

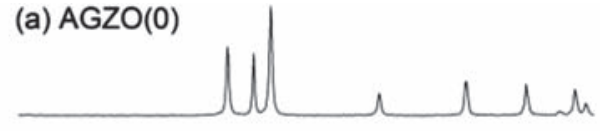

(b) $A G Z O(0.25)$
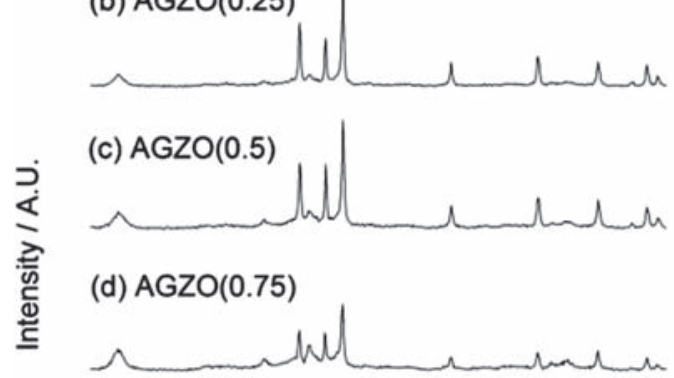

(e) $\triangle A G Z O(0.25)$

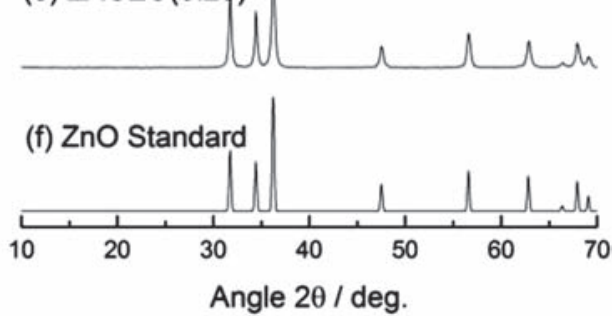

Figure 2. The pXRD patterns for as-prepared AGZO samples (a) $\operatorname{AGZO}(0)$, (b) $\operatorname{AGZO}(0.25)$, (c) $\operatorname{AGZO}(0.5)$ and (d) $\mathrm{AGZO}(0.75)$, where the number in brackets indicates the concentration of formic acid used in the precursor solution, (e) $\triangle \mathrm{AGZO}(0.25)$, formed by heat treatment of $\mathrm{AGZO}(0.25)$, and (f) the powder XRD pattern for the $\mathrm{ZnO}$ standard (PDF No. 01-076-0704). ${ }^{28}$ 
Table I. XRD pattern peak positions for heat-treated $\Delta \mathrm{AGZO}(0.25)$ and their assigned Miller indices.

\begin{tabular}{lc}
\hline $2 \theta /^{\circ}$ & Miller indices, $(h k l)$ \\
\hline 31.8 & $(100)$ \\
34.4 & $(002)$ \\
36.3 & $(101)$ \\
42.8 & $(102)$ \\
47.5 & $(110)$ \\
56.6 & $(103)$ \\
62.9 & $(200)$ \\
66.4 & $(112)$ \\
67.9 & $(201)$ \\
69.1 & $(004)$ \\
\hline
\end{tabular}

see Fig. 2(e)). ${ }^{28}$ Peak positions and assignments are shown in Table I. However, at least one minor impurity phase was also observed, with peaks at $2 \theta$ values of $12.9^{\circ}, 28.0^{\circ}$, $31.0^{\circ}$, and $32.8^{\circ}$ that could not be identified from the Bragg peaks present. $\mathrm{CHN}$ analysis on the as-prepared samples revealed increasing amounts of $\mathrm{C}, \mathrm{N}$, and $\mathrm{H}$ (2.12-2.99\%, $0-1.18 \%$ and $0.96-1.24 \%$, respectively) with increasing concentration of formic acid in the reaction mixture. As shown in Figure 2(e), upon heat treatment all impurities were removed in the XRD data, affording phasepure, crystalline wurtzite $\mathrm{ZnO}$ as shown by comparison to
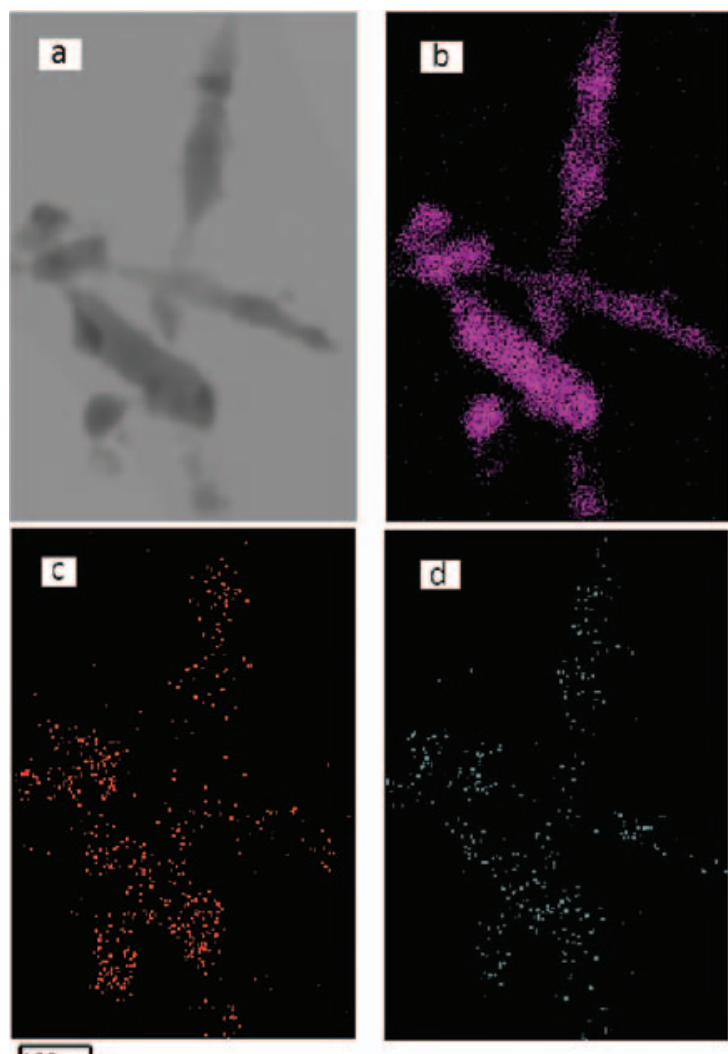

Figure 3. EDS elemental mapping of a scan for sample $\triangle \mathrm{AGZO}(0.25)$. The scanned image, and mappings of $\mathrm{Zn}, \mathrm{Al}$ and $\mathrm{Ga}$ are shown in panes (a-d) respectively. the $\mathrm{ZnO}$ reference pattern. ${ }^{28} \mathrm{CHN}$ analysis post-treatment showed negligible amounts of $\mathrm{C}, \mathrm{H}$, and $\mathrm{N}$, which may suggest that these were part of the unidentified impurity phase observed in the as-prepared XRD patterns.

To confirm the presence of both $\mathrm{Al}$ and $\mathrm{Ga}$ in the sample, energy-dispersive X-ray spectroscopy (EDS) (Fig. 3) and X-ray photoemission spectroscopy (XPS) (Fig. 4) were carried out. EDS mapping of $\mathrm{Zn}(2 p \mathrm{~K} \alpha), \operatorname{Al}(2 p$ $\mathrm{K} \alpha)$ and $\mathrm{Ga}(2 p \mathrm{~K} \alpha)$ emissions clearly showed these elements were homogenously distributed, on a length scale of approximately 1-2 $\mu \mathrm{m}$, throughout the $\mathrm{ZnO}$ nanoparticles. EDS indicated relative atomic proportions of $\mathrm{Zn}, \mathrm{Al}$ and Ga to be $94.6 \%, 3.9 \%$ and $1.5 \%$ respectively, which was comparable to the starting solution ratio of 95:3:2.

XPS analysis confirmed the presence of zinc, aluminium, and gallium within the heat-treated sample, with no other impurities observed within the survey spectrum. High resolution scans were carried out in the $\mathrm{Zn} 2 p, \mathrm{Al} 3 d$, $\mathrm{Ga} 2 p, \mathrm{O} 1 s$ and $\mathrm{C} 1 s$ regions of the spectrum. The binding energy scale for all measurements was calibrated using the adventitious $\mathrm{C} 1 s$ peak at $284.7 \mathrm{eV}$, and the peak positions are summarised in Table II. A set of doublet peaks at binding energies of $1021.5 \mathrm{eV}$ and $1044.6 \mathrm{eV}$ corresponded to the $2 p_{3 / 2}$ and $2 p_{1 / 2}$ levels of $\mathrm{Zn}^{2+}$, respectively. ${ }^{29} \mathrm{Sim}$ ilarly, the presence of $\mathrm{Al}^{3+}$ and $\mathrm{Ga}^{3+}$ was confirmed by the presence of peaks at binding energies of $74.1 \mathrm{eV}$ (Al $\left.2 p_{3 / 2}\right)^{30}$ and $1117.8 \mathrm{eV}\left(\mathrm{Ga} 2 p_{3 / 2}\right) \cdot{ }^{31}$ An additional set of peaks was observed in the $\mathrm{Al} 2 p$ region, at a binding energy of $76.4 \mathrm{eV}$ for $\mathrm{Al} 2 p_{3 / 2}$. Al $2 p$ peaks at this higher binding energy are typical of hydroxyl-species such as $\mathrm{AlO}(\mathrm{OH})$ and $\mathrm{Al}(\mathrm{OH})_{3},{ }^{32}$ likely to have resulted from hydration of near-surface Al-species within the sample. Analysis of the $\mathrm{O} 1 s$ region revealed a number of oxygencontaining species, attributable to the presence of multiple oxygen environments within the doped $\mathrm{ZnO}$ structure, as well as oxygen-containing surface contaminants. The presence of multiple $\mathrm{O} 1 s$ peaks prevented accurate deconvolution of the spectrum.

To investigate the influence of formic acid on the morphology of the particles, TEM was carried out on the asprepared samples, as shown for samples $\operatorname{AGZO}(0)$ and $\operatorname{AGZO}(0.25)$, which are shown in Figure 5. The TEM images showed similar shapes of particles, a mixture of small spheroids and larger rods being present in the absence of formic acid, whereas more, sharper rods were formed in the presence of formic acid.

To assess the electrical performance of the materials, Hall Effect measurements were carried out. Three measurements were made for each sample and the mean value calculated. Of the materials reported in this work, $\Delta \mathrm{AGZO}(0.25)(\Delta$ denoting heat treatment) showed the lowest resistivity of $4.62 \times 10^{-2} \Omega \mathrm{cm}( \pm 0.07 \times$ $10^{-2} \Omega \mathrm{cm}$ ), an excellent result for a pressed-nanopowder disc. Previous synthesis of ITO by the same CHFS process, processed and tested by similar methods, ${ }^{33}$ showed 

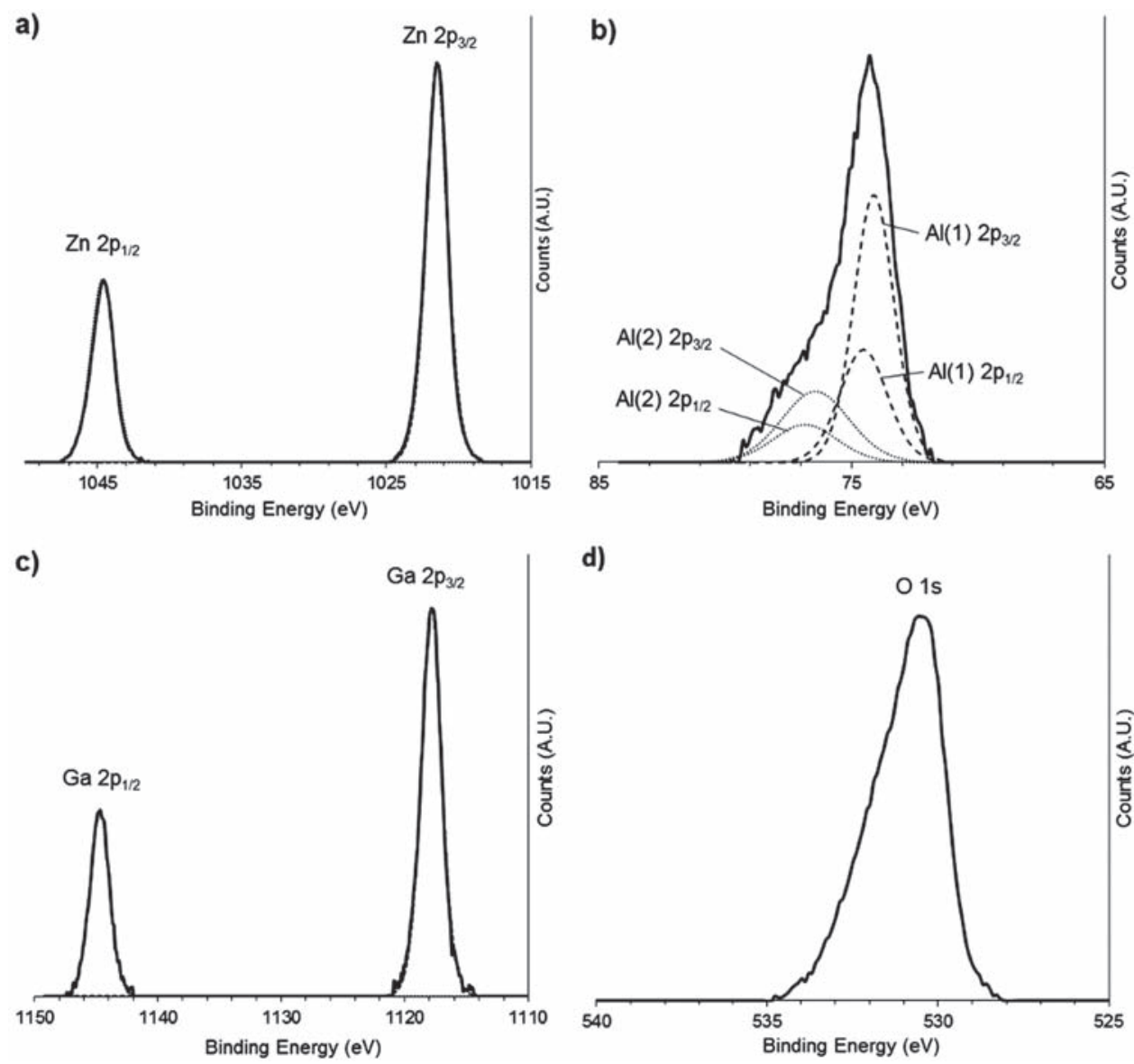

Figure 4. XPS spectra of $\triangle \mathrm{AGZO}(0.25)$, showing (a) Zn $2 p$ region; (b) $\mathrm{Al} 2 p$ region; (c) Ga $2 p$ region; and (d) O1s region. The solid black trace shows the recorded spectrum, whilst dotted traces show the fitted peaks.

resistivities as low as $6.0 \times 10^{-3} \Omega \mathrm{cm}$, less than an order of magnitude lower than those reported herein. Previous efforts based on pressed discs, also looking at ITO, yielded comparable results to that reported herein, e.g., $1-3 \times$ $10^{-2} \Omega \mathrm{cm}^{34,35}$

In summary, a CHFS process was used to synthesise AGZO, a cheap and sustainable alternative to ITO, as a transparent conducting oxide material. The measured resistivities were as low as $4.55 \times 10^{-2} \Omega \mathrm{cm}$ for a pressed nanopowder disc. Further study will investigate both the scale-up and optimization of the material composition and deposition of the nanopowders into thin films by various methods including inkjet printing and spin coating, which the authors believe will result in greatly improved

Table II. High resolution XPS binding energy positions for the metals $\left(2 p_{3 / 2}\right)$ in heat-treated $\triangle \mathrm{AGZO}(0.25)$.

\begin{tabular}{lc}
\hline Binding energy/eV & Assignment \\
\hline 74.1 & Al $2 p_{3 / 2}$ \\
76.4 & Al $2 p_{3 / 2}$ \\
1021.5 & Zn $2 p_{3 / 2}$ \\
1117.8 & Ga $2 p_{3 / 2}$ \\
\hline
\end{tabular}

resistivities for these underexplored materials. The results of these endeavors will be reported in due course.

\section{EXPERIMENTAL DETAILS}

The reagents used were potassium hydroxide (Fisher Scientific, Leicestershire, UK), zinc nitrate hexahydrate, $98 \%$ (Sigma Aldrich, Dorset, UK), aluminium nitrate nonahydrate, 98+\% (Sigma Aldrich, Dorset, UK), gallium nitrate hydrate, 99.999\% (Alfa Aesar, Lancashire, UK) and formic acid, $\geq 95 \%$ (Sigma Aldrich, Dorset, UK).

Deionised water was used throughout synthesis (resistivity $>10 \mathrm{M} \Omega \mathrm{cm}$ ). The pressure in the reactor was 24.1 $\mathrm{MPa}$ and the mixing temperature $335^{\circ} \mathrm{C}$. Hydrated zinc, aluminium, and gallium nitrate salts were dissolved as a single aqueous solution and used as the metal precursors at a total metal concentration of $0.2 \mathrm{M}$. Formic acid was added to the metal precursor solution at concentrations of $0.25-0.75 \mathrm{M}$. The reactor scheme is shown in Figure 1; the metal precursor solution was run through pump P2, $0.4 \mathrm{M} \mathrm{KOH}$ solution through P3, while P1 provided the supercritical water feed at flow rates of 40 , 40 , and $80 \mathrm{ml} \mathrm{min}{ }^{-1}$ respectively. AGZO nanoparticles were formed in flow in the mixer and cooled in flow 

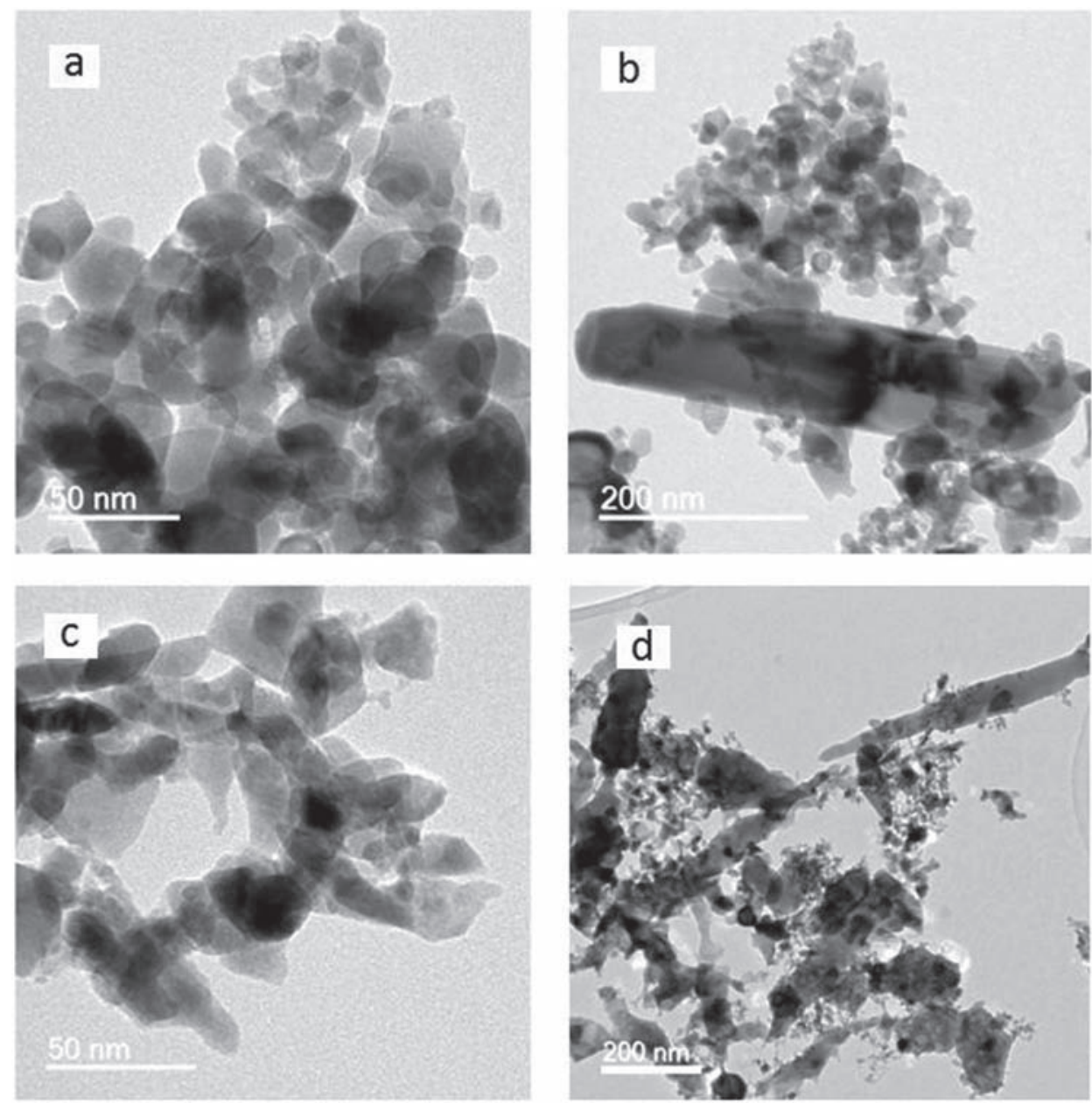

Figure 5. TEM images of untreated samples (a) $\mathrm{AGZO}(0)$, (b) $\mathrm{AGZO}(0)$, (c) $\mathrm{AGZO}(0.25)$, and (d) $\mathrm{AGZO}(0.25)$.

before being collected in an aqueous slurry after passing through a backpressure regulator (BPR, Tescom, Elk River, USA). The nanoparticle-laden slurries were washed with deionised water and the wet solids freeze-dried (Virtis Genesis $35 \mathrm{XL}$ ), resulting in freeflowing powders. Powder $\mathrm{X}$-ray diffraction patterns were collected using a Bruker D4 diffractometer, employing a copper source $\left(\mathrm{Cu} \mathrm{K}_{\alpha}\right.$, $\lambda=1.54 \AA$ ). Energy Dispersive X-ray Spectroscopy (EDS) was carried out using an Oxford Instruments X-Max ${ }^{\mathrm{N}} 80$ $\mathrm{T}$ Silicon Drift Detector (SDD) fitted to a Jeol $200 \mathrm{kV}$ transmission electron microscope and data processed using AZtec ${ }^{\circledR}$ software.

The nanopowders were pressed into compact discs (density $2.5 \mathrm{~g} \mathrm{~cm}^{-3}$ ) of thickness $0.9-1.0 \mathrm{~mm}$ under a force of 5 tons using a bench-top press. The discs were heat treated under $5 \% \mathrm{H}_{2} / \mathrm{N}_{2}$ at $500{ }^{\circ} \mathrm{C}$ for 3 hours. Hall Effect measurements were carried out using the van der Pauw method to determine the bulk resistivity of the materials. Four gold contacts were sputtered onto the heat-treated discs, which were then put under an input current of $1 \mathrm{~mA}$ and a calibrated magnetic field of $0.58 \mathrm{~T}$. The transverse voltage was then measured, and finally the measurement was repeated by reversing the direction of the magnetic field and the current.

Acknowledgments: The EPSRC is thanked for funding the project 'Sustainable Manufacturing of Transparent Conducting Oxide (TCO) Inks and Thin Films' (reference number EP/L017709/1). Claire Carmalt and Ivan Parkin are thanked for use of the Hall Probe.

\section{References and Notes}

1. D. S. Ginley and C. Bright, MRS Bull. 25, 15 (2000).

2. S. Ray, R. Banerjee, N. Basu, A. K. Batabyal, and A. K. Barua, J. Appl. Phys. 54, 3497 (1983).

3. H. Kim, C. M. Gilmore, A. Piqué, J. S. Horwitz, H. Mattoussi, H. Murata, and D. B. Chrisey, J. Appl. Phys. 86, 6451 (1999).

4. J. Ederth, P. Heszler, A. Hultåker, G. Niklasson, and C. Granqvist, Thin Solid Films 445, 199 (2003).

5. H. Bisht, H.-T. Eun, A. Mehrtens, and M. Aegerter, Thin Solid Films 351, 109 (1999).

6. K. Maki, N. Komiya, and A. Suzuki, Thin Solid Films 445, 224 (2003).

7. K. Ellmer, Nat. Photonics 6, 809 (2012).

8. Y. Liu, Y. Li, and H. Zeng, Journal of Nanomaterials 9, (ID 196521) (2013). 
9. Z. Ayadi, L. El Mir, K. Djessas, and S. Alaya, Mater. Sci. Eng., C 28,613 (2008).

10. H.-R. An, H.-J. Ahn, and J.-W. Park, Ceram. Int. 41, 2253 (2015).

11. T. Minami and T. Miyata, Thin Solid Films 517, 1474 (2008).

12. W. Lee, S. Shin, D.-R. Jung, J. Kim, C. Nahm, T. Moon, and B. Park, Current Applied Physics 12, 628 (2012).

13. J. Liu, W. Zhang, D. Song, Q. Ma, L. Zhang, H. Zhang, and H. Song, Ceram. Int. 40, 12905 (2014).

14. J.-H. Kang, M. H. Lee, D. W. Kim, Y. S. Lim, W.-S. Seo, and H.-J. Choi, Current Applied Physics 11, S333 (2011).

15. P. J. M. Isherwood, N. Neves, J. W. Bowers, P. Newbatt, and J. M. Walls, Thin Solid Films 566, 108 (2014).

16. J. Liu, W. Zhang, D. Song, Q. Ma, L. Zhang, H. Zhang, and H. Song, Ceram. Int. 40, 12905 (2014).

17. S.-C. Chang, Nanoscale Research Letters 9, 562 (2014).

18. J. Lu, K. Minami, S. Takami, M. Shibata, Y. Kaneko, and T. Adschiri, ACS Applied Materials and Interfaces 4, 351 (2012).

19. J. B. M. Goodall, S. Kellici, D. Illsley, R. Lines, J. C. Knowles, and J. A. Darr, RSC Advances 4, 31799 (2014).

20. A. J. T. Naik, R. I. Gruar, C. J. Tighe, I. P. Parkin, J. A. Darr, and R. Binions, Sensors and Actuators B: Chemical (2014), doi:10.1016/j.snb.2014.10.039.

21. C. J. Tighe, R. Q. Cabrera, R. I. Gruar, and J. A. Darr, Ind. Eng. Chem. Res. 52, 5522 (2013).
22. J. B. M. Goodall, D. Illsley, R. Lines, N. M. Makwana, and J. A. Darr, ACS Combinatorial Science 17, 100 (2015).

23. R. I. Gruar, C. J. Tighe, and J. A. Darr, Ind. Eng. Chem. Res. 52, 5270 (2013).

24. M. D. McCluskey and S. J. Jokela, Physica B: Condensed Matter 401-402, 355 (2007)

25. T. Yagasaki, S. Saito, and I. Ohmine, J. Chem. Phys. 117, 7631 (2002).

26. J. Yu and P. E. Savage, Ind. Eng. Chem. Res. 37, 2 (1998)

27. Y. Zhang, J. Zhang, L. Zhao, and C. Sheng, Energy Fuels 24, 95 (2010).

28. H. Schulz and K. H. Thiemann, Solid State Commun. 32, 783 (1979).

29. L. G. Mar, P. Y. Timbrell, and R. N. Lamb, Thin Solid Films 223, 341 (1993).

30. J. A. Kovacich and D. Lichtman, J. Electron. Spectrosc. Relat. Phenom. 18, 341 (1980).

31. G. Schoen, Journal of Electron Spectroscopy Related Phenomena 2, 75 (1973).

32. T. Gougousi, D. Barua, E. Young, and G. Parsons, Chem. Mater. 17, 5093 (2005).

33. P. Marchand, N. M. Makwana, C. J. Tighe, R. I. Gruar, I. P. Parkin, C. J. Carmalt, and J. A. Darr, Under Submission (2015).

34. G. Bühler, D. Thölmann, and C. Feldmann, Adv. Mater. 19, 2224 (2007).

35. E. Hammarberg, A. Prodi-Schwab, and C. Feldmann, Thin Solid Films 516, 7437 (2008)

Received: 30 June 2015. Accepted: 23 September 2015. 